\title{
MULTIPLE-ANTENNA CDMA MOBILE RECEPTION USING CONSTRAINED NORMALIZED ADAPTIVE ALGORITHMS
}

\author{
Stefan Werner ${ }^{1}$, J. A. Apolinário Jr. ${ }^{2}$, and Timo I. Laakso ${ }^{1}$ \\ ${ }^{1}$ Helsinki University of Technology \\ Laboratory of Telecommunications Technology \\ P.O. Box 3000 \\ 02015 HUT, FINLAND \\ E-mail: Stefan.Werner@hut.fi, Timo.Laakso@hut.fi \\ phone: +35894512437 fax: +35894512474 \\ ${ }^{2}$ Instituto Militar de Engenharia \\ Departamento de Engenharia Elétrica \\ Praça Gal. Tibúrcio, 80 \\ 22.290-270, Rio de Janeiro, BRAZIL \\ E-mail: apolin@aquarius.ime.eb.br \\ phone and fax: +55 215420999
}

\begin{abstract}
In this paper we analyze adaptive algorithms for a multiple-antenna Code Division Multiple Access (CDMA) mobile receiver. We study the constrained versions of the Binormalized DataReusing LMS (BNDR-LMS) and the Normalized LMS (NLMS). Their performance is compared with that of the classical Frost algorithm. Time-varying stepsequences are used to further enhance the performance of the receiver. The results show that considerable improvement in the convergence speed can be achieved with the use of the normalized algorithms compared with the conventional Frost algorithm. Using an optimal step-sequence in the update provides a fast convergence and a nearoptimum steady-state performance.
\end{abstract}

\section{INTRODUCTION}

Direct-Sequence CDMA is a very strong candidate for the technique for the future $3^{\text {rd }}$-generation or Universal Mobile Telecommunication System (UMTS). Development of dedicated adaptive algorithms that provide good and reliable performance in the harsh radio channel environment is thus of increasing importance.

In CDMA reception at the mobile end there are several special requirements. The resources for processing are severely constrained by the physical size of the receiver and the strict limitations for power consumption. Furthermore, the other users' codes are not necessarily known at the mobile receiver and the estimation of other users' channel parameters may especially involve too complex processing for the mobile resources. This implies that detection algorithms based on simultaneous optimal detection of multiple users [1] or its suboptimal approximations [2], [3] may have to be abandoned. Hence, the multiple-access interference (MAI) has to be suppressed by other techniques.

A class of algorithms suitable for mobile reception are blind interference cancellers which do not assume knowledge of other users' codes or their channel parameters. This kind of algorithms have been applied to CDMA reception in [4]-[8]. In [4] a blind LMS-type of algorithm was developed for interference suppression in a CDMA system. The algorithm which is similar to the gradient projection algorithm [9] suffers from the same problems as the conventional LMS algorithm, i.e., slow convergence. Furthermore there is no inherent mechanism preventing accumulation of roundoff errors resulting in that the receiver drifts away from the constraint hyper-plane. The problem with error accumulation can be taken care of by using the Frost algorithm [9] which is a simple LMS-type of algorithm ensuring the constraints in every iteration.

The Frost algorithm was applied to a CDMA mobile receiver equipped with multiple antennas in [5]. To speed up the convergence speed the use of normalized algorithms, like the NLMS [10] and the BNDR-LMS [11] algorithms, can be used. Normalized algorithms often achieve a faster convergence than the conventional LMS at the expense of low increase in the complexity. Another approach is to use leastsquares techniques [12] to improve the performance. In a mobile unit it is desirable to keep the signal processing as simple as possible due to complexity 
constraints. In this paper we develop two normalized LMS-type of algorithms suitable for a multipleantenna mobile CDMA receiver and compare those to the Frost algorithm. The receiver requires knowledge of the spreading waveforms in every antenna and can be seen as a generalization of [4] to include multiple antennas.

This paper is organized as follows. Section II briefly describes the signal model. The multiple-antenna receiver is derived in Section III. In Section IV, the adaptive algorithms used are considered. The performance of the receiver and its adaptive implementations is demonstrated with a simplified example in Section V, followed by conclusions.

\section{SIGNAL MODEL}

In downlink transmission (base station to mobile), signals associated with a number of simultaneously active users are transmitted over the same mobile channel. A mobile receiver, equipped with $N$ antennas, will receive the transmitted signal over $N$ different channels.

The system under consideration consists of $K$ users transmitting information with binary antipodal signals with bit duration $T_{b}$. We assume an AWGN channel but an extension to more realistic fading multipath channels is straightforward.

The continuous transmitted signal is formed by:

$$
x(t)=\sum_{n=-\infty}^{\infty} \sum_{i=1}^{K} A_{i} b_{i}(n) s_{i}\left(t-n T_{b}\right)
$$

where for the $k^{\text {th }}$ user, $b_{k}(m) \in\{-1,1\}$ is the $m^{\text {th }}$ bit, $A_{k}$ is the relative amplitude due to power control, $s_{k}(t)$ is signature sequence (code) with $G=T_{b} / T_{c}$ number of chips per bit, $\omega_{c}$ is the carrier frequency and $\phi$ is the carrier phase.

In front of every antenna is down-converter followed by a chip-matched filter (integrate and dump filter with integration time $T_{c}$ ) as can be seen in Fig. 1.

If the samples from the received sequence during the $m^{\text {th }}$ bit interval are collected in the vectors we can write the received discrete-time signal as:

$$
\mathbf{r}_{i}(m)=\mathbf{S}_{i} \mathbf{A}_{i} \mathbf{b}(m)+\mathbf{n}_{i}(m)
$$

where $\mathbf{S}_{i}$ is the $G \times K$ spreading matrix containing the spreading sequences for the different users:

$$
\mathbf{S}_{i}=\left[\mathbf{S}_{1 i} \mathbf{S}_{2 i} \ldots \mathbf{S}_{k, i}\right]
$$

where $\mathbf{S}_{k, i}$ is the discrete-time delayed version of the $k^{\text {th }}$ user's sampled code sequence at antenna $i, \mathbf{A}_{i}$ is a diagonal amplitude matrix of the form:

$$
\mathbf{A}_{i}=\operatorname{diag}\left[a_{i} A_{1} a_{i} A_{2} \cdots a_{i} A_{k}\right]
$$

where $a_{i}=e^{j(\phi-\omega \tau)}$ is the complex phase factor at the $i^{\text {th }}$ antenna. $\mathbf{b}(m)$ is a vector containing the transmitted bits of the users:

$$
\mathbf{b}(m)=\left[b_{1}(m) b_{2}(m) \ldots b_{K}(m)\right]^{\mathrm{T}}
$$

Finally, $\mathbf{n}_{i}(m)$ is a vector of the noise components of the antenna elements. These noise components are assumed to be independent, i.e.,

$$
E\left\{\mathbf{n}_{i}(m) \mathbf{n}_{j}^{\mathrm{H}}(m)\right\}=\sigma_{i}^{2} \mathbf{I}_{G} \delta(i-j)
$$

If the antennas are spaced close together, the sampled code sequences will be practically the same in all antennas. In the case of an $\mathrm{N}$-element linear array, the phase factor is simply given by

$$
a_{i}=\exp \left[j\left(\phi-2 \pi(i-1) \frac{\Delta}{\lambda_{c}} \sin \theta\right)\right]
$$

where $\Delta$ is the element spacing, $\lambda_{c}$ is the wavelength of the carrier and $\theta$ is the direction of the incoming signal with respect to the array normal.

\section{MULTIPLE-ANTENNA RECEIVER}

In this section, a linear single-user multi-antenna (LSUMA) detector is derived. The detector has $N$ antennas and assumes knowledge of the phase factors in every antenna. The structure of the receiver is shown in Fig. 1. Each of the $N$ antenna branches contains a linear filter whose coefficients are to be optimized. The filtered signals from each antenna are then added together to form a decision variable. In Fig. 1, $\mathbf{r}_{i}$ denotes the received signal after chipmatched filtering at antenna $i, \mathbf{h}_{i}$ contains the complex filter coefficients for the $i^{\text {th }}$ antenna, and $z$ is the decision variable formed by adding the filtered outputs from each antenna.

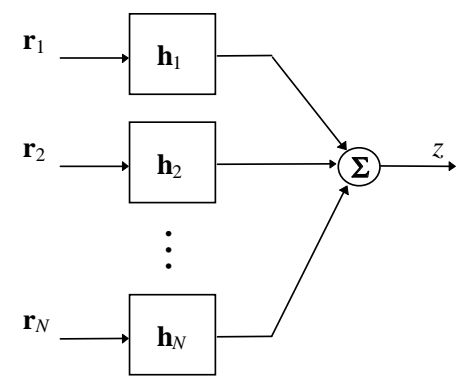

Figure 1: Structure of linear detector 
In order to get a compact notation, let us collect the filter coefficients and the received sequences from the antennas in vectors as

$$
\begin{gathered}
\mathbf{h}=\left[\begin{array}{lll}
\mathbf{h}_{1}^{\mathrm{T}} & \ldots & \mathbf{h}_{N}^{\mathrm{T}}
\end{array}\right]^{\mathrm{T}} \\
\mathbf{r}=\left[\begin{array}{llll}
\mathbf{r}_{1}^{\mathrm{T}} & \mathbf{r}_{2}^{\mathrm{T}} & \ldots & \mathbf{r}_{N}^{\mathrm{T}}
\end{array}\right]^{\mathrm{T}}
\end{gathered}
$$

Using the above notation, the output from the receiver can be written as

$$
z=\mathbf{h}^{\mathrm{H}} \mathbf{r}
$$

The variance of the output, i.e., the output power, is

$$
E\left\{|z|^{2}\right\}=E\left\{\mathbf{h}^{\mathrm{H}} \mathbf{r r}^{\mathrm{H}} \mathbf{h}\right\}=\mathbf{h}^{\mathrm{H}} \mathbf{R} \mathbf{h}
$$

where $\mathbf{R}$ is the correlation matrix.

The filter coefficients $\mathbf{h}$ are found by minimizing the output variance of (11) under the constraints that the desired user's code sequence in every antenna can pass with unity response. To get a compact form we introduce the $G N \times N$ matrix $\mathbf{C}$ and the $N \times 1$ vector $\mathbf{u}$ as

$$
\begin{gathered}
\mathbf{C}=\left[\begin{array}{cccc}
a_{1} \mathbf{s}_{1,1} & 0 & \ldots & 0 \\
0 & a_{2} \mathbf{s}_{1,2} & 0 & \vdots \\
\vdots & 0 & \ddots & 0 \\
0 & \ldots & 0 & a_{N} \mathbf{s}_{1, N}
\end{array}\right] \\
\mathbf{u}=\left[\left|a_{1}\right|^{2}\left|a_{2}\right|^{2} \ldots\left|a_{N}\right|^{2}\right]^{\mathrm{T}}
\end{gathered}
$$

The minimization problem can now be formulated as

$$
\begin{aligned}
& \hat{\mathbf{h}}=\arg \min _{\mathbf{h}} E\left\{|z|^{2}\right\} \\
& \text { subject to: } \quad \mathbf{C}^{\mathrm{H}} \mathbf{h}=\mathbf{u}
\end{aligned}
$$

The solution to this problem is found by the method of Lagrange multipliers

$$
\mathbf{h}_{\mathrm{opt}}=\mathbf{R}^{-1} \mathbf{C}\left[\mathbf{C}^{\mathrm{H}} \mathbf{R}^{-1} \mathbf{C}\right]^{-1} \mathbf{u}
$$

The minimum output variance is obtained by substituting (15) into (11):

$$
E\left\{|z|^{2}\right\}=\mathbf{u}^{\mathrm{H}}\left[\mathbf{C}^{\mathrm{H}} \mathbf{R}^{-1} \mathbf{C}\right]^{-1} \mathbf{u}
$$

The closed-form solution is not suitable for practical implementation, as we need to estimate the correlation matrix and perform a matrix inversion. In the next section we consider adaptive implementations of the detector.

\section{ADAPTIVE ALGORITHMS}

In this section, we study three adaptive implementations of Eq. (15). The algorithms are: the
Frost algorithm, and the constrained versions of the NLMS and the BNDR-LMS algorithms [6]. The Frost algorithm is simply the constrained version of the conventional LMS algorithm. The BNDR-LMS update performs normalization onto two orthogonal directions obtained from consecutive data pairs within each iteration as compared to the NLMS which only does it for one direction.

All the algorithms make use of a projection matrix $\mathbf{P}=\mathbf{I}-\mathbf{C}\left(\mathbf{C}^{\mathrm{H}} \mathbf{C}\right)^{-1} \mathbf{C}^{\mathrm{H}}$ and a fixed vector $\mathbf{f}=\mathbf{C}\left(\mathbf{C}^{\mathrm{H}} \mathbf{C}\right)^{-1} \mathbf{u}$. The projection matrix $\mathbf{P}$ removes all components perpendicular to the plane $\mathbf{C}^{\mathrm{H}} \mathbf{h}=0$ and the vector $\mathbf{f}$ moves the solution back onto the constraint plane $\mathbf{C}^{\mathrm{H}} \mathbf{h}=\mathbf{u}$. For detailed derivations of the algorithms see [6], [9].

The Frost algorithm is given by

$$
\left.\mathbf{h}_{\mathrm{F}}(m+1)=\mathbf{P}\left[\mathbf{h}_{\mathrm{F}}(m)-\mu z^{*}(m) \mathbf{r}(m)\right)\right]+\mathbf{f}
$$

Notice that the expression inside the brackets of Eq. (17) is the unconstrained LMS algorithm (reference signal set to zero).

The constrained NLMS is given by

$$
\begin{aligned}
\mathbf{h}_{\mathrm{N}}(m+1) & \left.=\mathbf{P}\left[\mathbf{h}_{\mathrm{N}}(m)-\mu \frac{z^{*}(m)}{\mathbf{r}^{\mathrm{H}}(m) \operatorname{Pr}(m)} \mathbf{r}(m)\right)\right] \\
& +\mathbf{f}
\end{aligned}
$$

The expression inside the brackets is similar to the unconstrained NLMS algorithm apart from the introduction of the projection matrix in the denominator of the second term. However, by using the property of $\mathbf{P}^{\mathrm{H}} \mathbf{P}=\mathbf{P}$ we can introduce the rotated vector $\mathbf{r}^{\prime}=\mathbf{P r}$ and the expression inside the brackets in Eq. (18) is just the unconstrained NLMS update for the rotated vector $\mathbf{r}^{\prime}$.

The constrained BNDR-LMS algorithm is given by

$$
\begin{gathered}
\mathbf{h}_{\mathrm{B}}(m+1)=\mathbf{P}\left[\mathbf{h}_{\mathrm{B}}(m)+\mu_{1 k} \mathbf{r}(m)+\mu_{2 k} \mathbf{r}(m-1)\right] \\
+\mathbf{f} \\
\mu_{1 k}=\frac{e_{1} \mathbf{r}^{\mathrm{H}}(m-1) \operatorname{Pr}(m-1)-e_{2} \mathbf{r}^{\mathrm{H}}(m-1) \mathbf{P r}(m)}{d e n} \\
\mu_{2 k}=\frac{e_{1} \mathbf{r}^{\mathrm{H}}(m) \mathbf{P r}(m)-e_{2} \mathbf{r}^{\mathrm{H}}(m-1) \operatorname{Pr}(m)}{d e n} \\
d e n=\mathbf{r}^{\mathrm{H}}(m) \mathbf{P r}(m) \mathbf{r}^{\mathrm{H}}(m-1) \operatorname{Pr}(m-1) \\
-\left|\mathbf{r}^{\mathrm{H}}(m-1) \mathbf{P r}(m)\right|^{2} \\
e_{1}=-\mathbf{r}^{\mathrm{H}}(m) \mathbf{h}_{B}(m)
\end{gathered}
$$




$$
e_{2}=-\mathbf{r}^{\mathrm{H}}(m-1) \mathbf{h}_{B}(m)
$$

Also here the expression inside the brackets of Eq. (19) is the unconstrained BNDR-LMS update for the rotated vector $\mathbf{r}^{\prime}$.

All the algorithms are initialized with $\mathbf{h}(0)=\mathbf{f}$. It is easy to check that the initial value satisfies the constraints in Eq. (14).

Fig. 2 depicts the coefficient vector update of the constrained NLMS algorithm. Note that $\mathbf{C}^{\mathrm{H}} \mathbf{h}=\mathbf{u}$ is the constraint plane and that the unconstrained NLMS algorithm takes a normalized step towards the plane $\mathbf{r}^{\mathrm{H}}(\mathrm{m}) \mathbf{h}=\mathbf{0}$ since the reference signal in this case is zero.

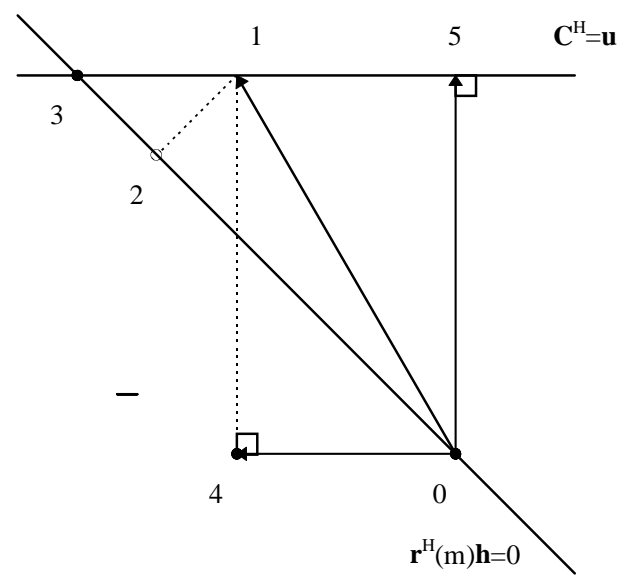

Figure 2: Coefficient vector update of the constrained NLMS algorithm :

(0) origin

(1) $\mathbf{h}(m)$

(2) $\mathbf{h}(m+1)$ of the unconstrained NLMS algorithm

(3) $\mathbf{h}(m+1)$ of the constrained NLMS algorithm

(4) $\mathbf{P h}(m)$

(5) $\mathbf{f}=\mathbf{C}\left(\mathbf{C}^{\mathrm{H}} \mathbf{C}\right)^{-1} \mathbf{u}=(\mathbf{I}-\mathbf{P}) \mathbf{u}$

\section{NUMERICAL RESULTS}

In this section, the receiver algorithms are simulated and their performance is compared. The antennas are structured as a uniform linear array (ULA) with spacing of half the wavelength and the direction of arrival is set to $15^{\circ}$. The system used in the example consists of 5 users with spreading sequences taken as Gold codes of length 7 . The signal-to-noise ratio for the desired user was set to $8 \mathrm{~dB}$ (in the absence of MAI). The simulation is carried out for one and two antennas.

In order to achieve fast convergence and small misadjustment we use time-varying step-size. The step-sizes correspond to the optimal sequences presented in [13].

As the performance measure we use the signal-tointerference ratio (SIR) at the output of the receiver which are presented in Figs. 3-4 for the Frost, NLMS, and BNDR-LMS as a function of the number of iterations. All the results are averaged over 500 runs. In order to be able to compare the algorithms, the Frost algorithm is plotted for two different step-sizes, one that results in a steady-state value comparable to the normalized algorithms and another that gives faster convergence.

In the plots the horizontal dashed line shows the optimum SIR value and the solid line corresponds to the conventional matched filter solution.

Fig. 3 show the results for one antenna. The step-sizes used in the Frost algorithm are: $\mu=2 \cdot 10^{-3}$ and $\mu=10 \cdot 10$

From Fig. 3 we can see that the performance of the NLMS and the BNDR-LMS algorithms is about the same but has considerable faster convergence than that of the Frost algorithm. It is of course possible to speed up the Frost algorithm even more by choosing a larger step-size. However, in order to have a convergence speed close to the normalized algorithms will result in a very large misadjustment.

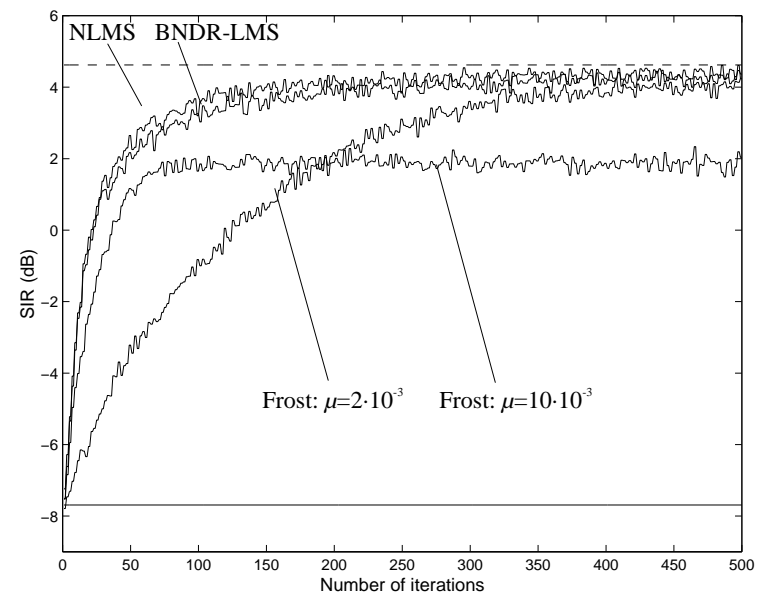

Figure 3: SIR as a function of the number of iterations for the algorithms in a system with one antenna and five users. The interfering users transmit at $10 \mathrm{~dB}$ higher power than the desired user.

In Fig. 4, the results for two antennas are plotted. The step-sizes used in the Frost algorithm are: $\mu=1 \cdot 10^{-3}$ and $\mu=5 \cdot 10^{-3}$.

As can be seen from Fig. 4, the normalized algorithms can still provide a convergence speed 
superior to that of the Frost algorithm. We can also see here that the misadjustment becomes higher as the step-size in the Frost algorithm increases.

In fact, the misadjustment is so high when using $\mu=5 \cdot 10^{-3}$ that the resulting steady-state is slightly lower than for the NLMS and BNDR-LMS using only one antenna. Furthermore, the convergence speed is about the same. As a consequence, the same performance in terms of convergence speed and steady-state value is achieved by only using one antenna and the normalized algorithms instead of using two antennas and the Frost algorithm.

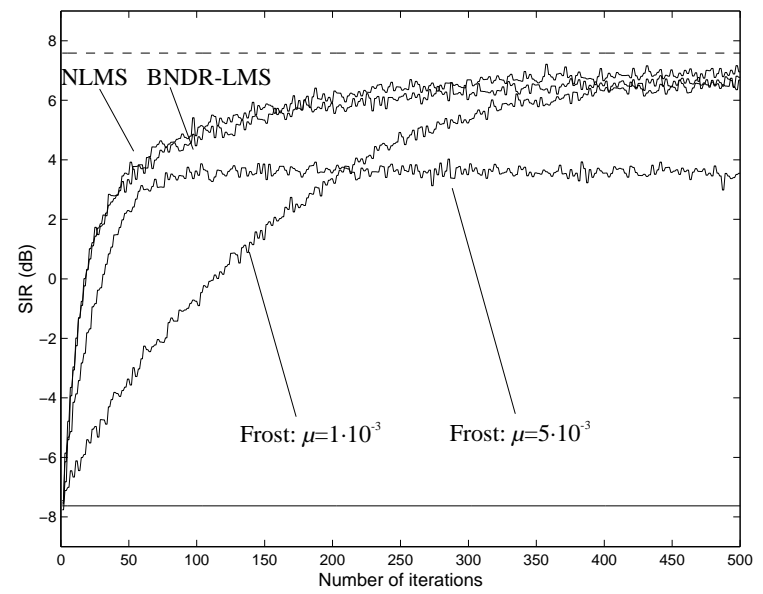

Figure 4: SIR as a function of the number of iterations for the algorithms in a system with two antennas and five users. The interfering users transmit at $10 \mathrm{~dB}$ higher power than the desired user.

\section{CONCLUSIONS}

In this paper, we have presented and compared three adaptive algorithms suitable for a DS-CDMA multiantenna receiver. The results showed that constrained versions of the normalized algorithms outperforms the conventional Frost algorithm in terms of both convergence speed and misadjustment.

\section{ACKNOWLEDGMENT}

This work is part of a research project of the Institute of Radio Communication (IRC) funded by Technology Development Center (TEKES), NOKIA Research Center, Telecom Finland and the Helsinki Telephone Company.

\section{REFERENCES}

[1] S. Verdú, "Minimum probability of error for asynchronous Gaussian multiple-access channels," IEEE Transactions on Information Theory, vol. IT32, pp. 85-96, Jan. 1986.

[2] M. K. Varansi and B. Aazhang, "Multistage detection in asynchronous code-division multipleaccess communications," IEEE Transactions on Communications, vol. 38, pp. 509-519, Apr. 1990.

[3] M. K. Varansi and B. Aazhang, "Near-optimum detection in synchronous code-division multipleaccess systems," IEEE Transactions on Communications, vol. 39, pp. 725-736, May 1991.

[4] M. Honig, U. Madhow, S. Verdú, "Blind adaptive multiuser detection", IEEE Transactions on Information Theory, vol. 41, pp. 944-960, Jul 1995.

[5] S. Werner, T. Laakso, J. Lilleberg, "Multiple-antenna receiver for CDMA mobile reception," International Conference on Communications, June 7-11 1998, Atlanta, Georgia, USA.

[6] J.A. Apolinario Jr., S. Werner, T. Laakso, P.S.R Diniz, "Constrained normalized adaptive filtering for CDMA mobile communications," Accepted to the European Signal Processing Conference, Sept 8-11, 1998, Island of Rhodes, Greece.

[7] J. B. Schodorf, D. B. Williams, "A blind adaptive interference cancellation scheme for CDMA systems", Proc. Asilomar Conf. Sig., Sys. and Comp., 1995.

[8] S. C. Park and J. F. Doherty, "Generalized projection algorithm for blind interference suppression in DS/CDMA communications," IEEE Transactions on Circuits and Systems, vol. 44,no. 6, pp. 453-460, June 1997.

[9] O. L. Frost, III, "An algorithm for linearly constrained adaptive array processing", Proceedings of IEEE, vol. 60, pp. 926-935, Aug 1972.

[10] P. S. R. Diniz, Adaptive Filtering: Algorithms and Practical Implementations, Kluwer Academic Publishers, Boston, MA, USA, 1997.

[11] J. A. Apolinário Jr., M. L. R. de Campos, P. S. R. Diniz, "The binormalized data-reusing LMS algorithm," Proceedings of the XV Simpósio Brasileiro de Telecomunicações, Recife, Brazil, 1997.

[12] L. S. Resende, J. M. T. Romano, and M. G. Bellanger, "A fast least-squares algorithm for linearly constrained adaptive filtering," IEEE Transactions on Signal Processing, vol. 44, pp. 1168-1174, May 1996.

[13] J.A. Apolinario Jr., P.S.R Diniz, T. Laakso, M. L. R. de Campos, "Step-size optimization of the BNDRLMS algorithm," Accepted to the European Signal Processing Conference, Sept 8-11, 1998, Island of Rhodes, Greece. 